\title{
Innovative $x$-ray polarimeters enable development of new NASA observatory
}

\section{Kevin Black}

Highly sensitive $x$-ray polarimeters will allow a small orbiting astronomical observatory to provide new insights into the twisted space around spinning black holes.

The polarization of x-rays emitted from black holes and neutron stars contains key astrophysical information that is inaccessible through either imaging, spectroscopy, or photometry. Yet, $\mathrm{x}$-ray polarimetry remains unexploited, largely because previously available polarimeters (based on x-ray scattering) lacked in sensitivity. In principle, the photoelectric effect provides a much more powerful basis for x-ray polarimetry. Until recently, however, daunting technical challenges prevented realization of a practical photoelectric device.

In the last decade, advances in micropattern gas detectorsvery finely spaced arrays of proportional counters-made astronomical x-ray polarimetry a possibility and fostered renewed interest in its prospects for scientific discovery. ${ }^{1-3}$ These instruments measure polarization by imaging the tracks of photoelectrons created as a result of x-ray absorption by the ambient gas. A given x-ray's polarization state is determined by the initial direction of the resulting photoelectron. While these detectors are orders of magnitude more sensitive than previously possible, their quantum efficiencies are still limited. Further improvements are required to place the most compelling polarization measurements-such as observations of supermassive black holes in distant galaxies-within the reach of a small spacebased observatory.

We are developing 'time-projection' polarimeters, which overcome the quantum-efficiency limitation by employing a different detector geometry and readout scheme. Our polarimeters also use a micropattern gas detector to image photoelectron tracks. However, instead of imaging by direct readout of a pixel array, we use a time-projection technique to create virtual pixels from a $1 \mathrm{D}$ array of strips (see Figure 1). ${ }^{4}$ This encoding scheme

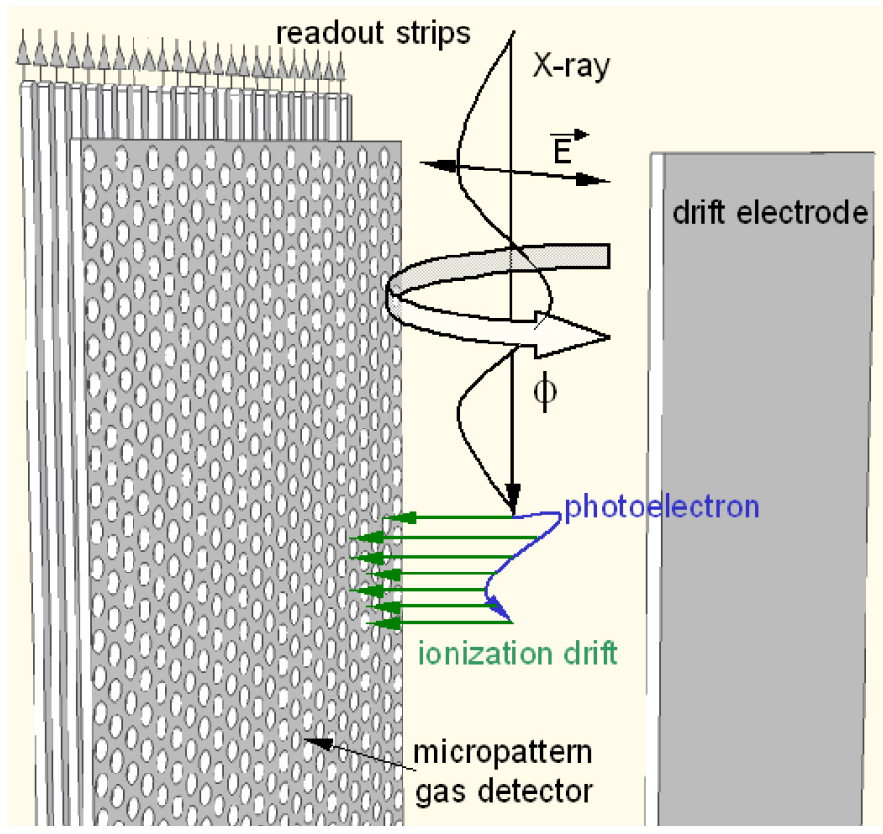

Figure 1. Time-projection polarimeter concept. Photoelectrons are emitted preferentially in the direction of the electric field $(\vec{E})$ with a $\sin ^{2} \theta \cos ^{2} \phi$ distribution, where $\phi$ is the polarization-dependent angle. The ionization trail left by the photoelectron drifts (in a uniform electric field) to the micropattern detector. An image is formed in the plane normal to the $x$-ray beam by binning the detected ionization by strip location and arrival time.

arranges detection elements alongside the incoming x-ray beam, which allows indefinite increases of the active depth (and hence the quantum efficiency) without loss of image resolution.

The relative simplicity of the instrument is reflected in our first demonstration detector, which required no new technology development and was fabricated entirely from off-the-shelf 


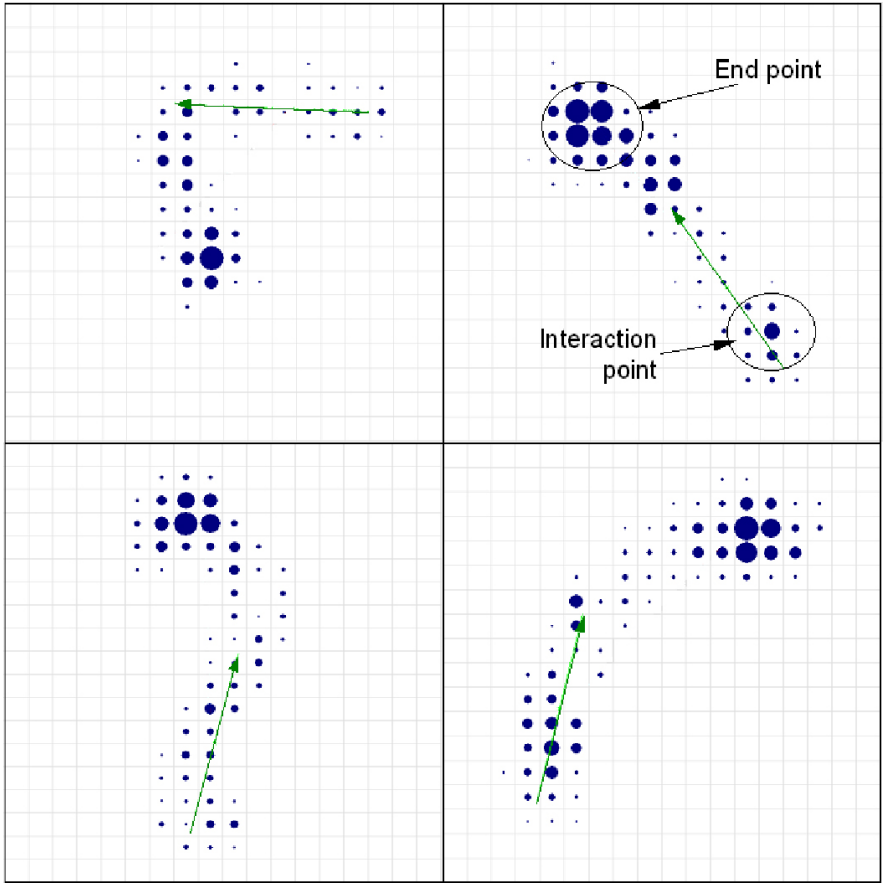

Figure 2. Images of photoelectron tracks from $5.9 \mathrm{keV} x$-rays in a timeprojection polarimeter. The pixels are on a $120 \mu \mathrm{m}$ spacing. The size of the dots reflects the amount of charge in each pixel. The green arrow shows the reconstructed emission direction.

components. Our device demonstrated a polarization response comparable to pixel-proportional counters, but with significantly greater quantum efficiency. ${ }^{5}$ More recently, we have been developing time-projection polarimeters suitable for spaceflight and capable of detecting polarization from faint astrophysical sources at the $1 \%$ level.

Our first flight-like polarimeter features highly stable micropattern gas detectors ${ }^{6}$ and an analog memory-based readout using a low-noise, low-power, radiation-hard, application-specific integrated circuit. ${ }^{7}$ Selected results from our prototype polarimeter are shown in Figures 2 and 3. An important aspect of achieving high sensitivity is ensuring that we can control systematic errors because they can create false polarization signals. Tests with unpolarized $x$-rays show no false signal below the $1 \%$ detection level.

We are beginning construction of the first prototype timeprojection polarimeters specifically for NASA's recently announced Gravity and Extreme Magnetism Small Explorer (GEMS), scheduled for launch in 2014. Its scientific goals require high-sensitivity polarization measurements in the $1-10 \mathrm{keV}$ energy band. The instrument will consist of three $30 \mathrm{~cm}$-deep polarimeters, each at the focus of a grazing-incidence $x$-ray

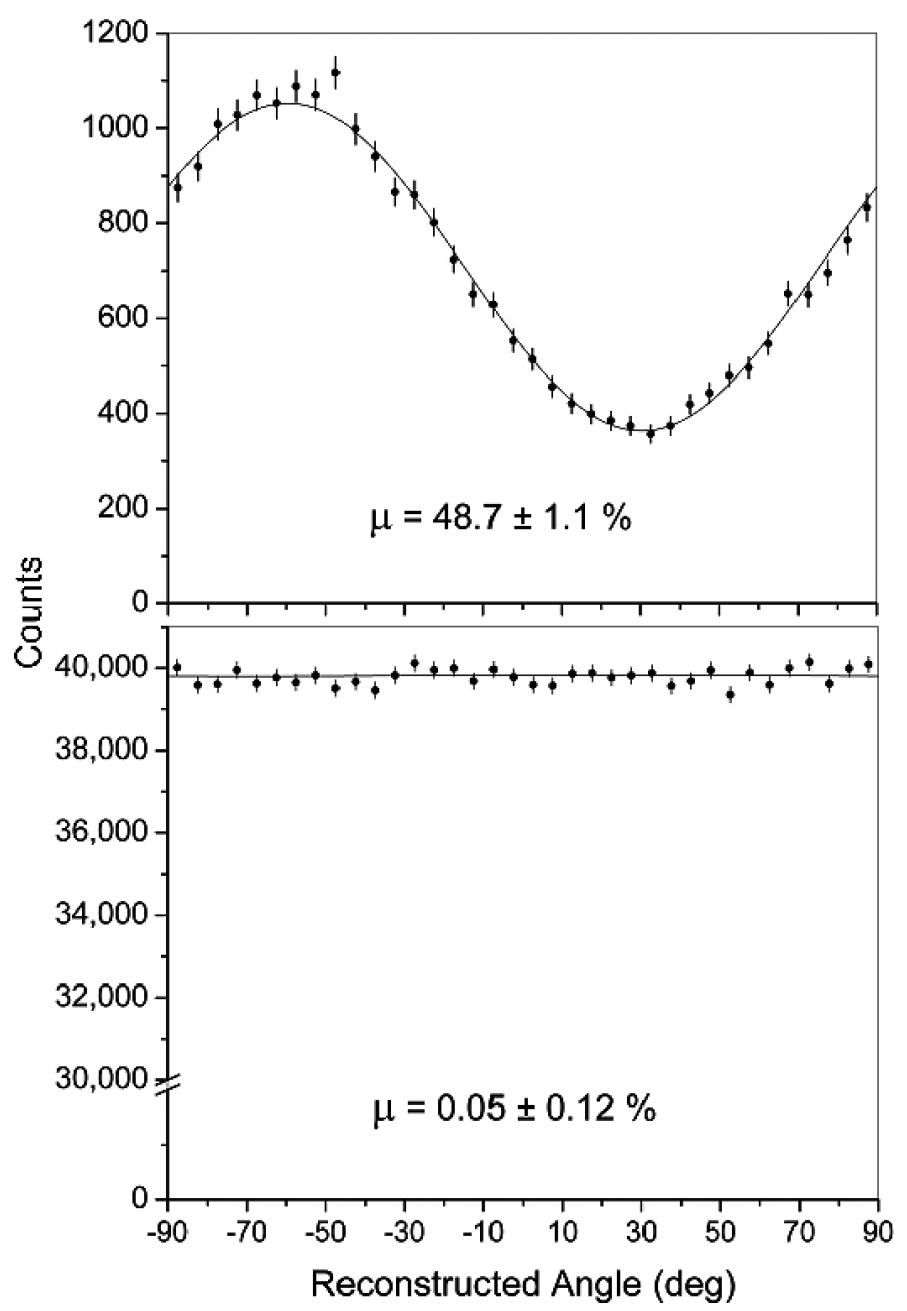

Figure 3. Reconstructed emission angles for polarized $4.5 \mathrm{keV} x$-rays (top) and unpolarized $5.9 \mathrm{keV} x$-rays (bottom). The lines are fits to the expected functional form, $A+B \cos ^{2} \phi$. The modulation factor, $\mu$, is a measure of the amplitude of the response. The polarized data shows a clear response, while the unpolarized data is consistent with no detected signal below the $1 \%$ level.

telescope similar to those used in current-generation x-ray observatories. ${ }^{8}$ We are also developing large-volume timeprojection polarimeters that could enable x-ray polarization measurements of gamma-ray bursts or solar flares.

With simple yet innovative new photoelectric time-projection x-ray polarimeters, the GEMS mission will open a new window on the universe. GEMS will achieve levels of x-ray-polarization sensitivity previously unattainable even with large observatories. The mission is therefore expected to answer fundamental 
questions about black holes, neutron stars, and their associated extreme gravitational and magnetic fields.

This article is on behalf of the $x$-ray polarimeter development group at NASA's Goddard Space Flight Center, which also includes R. G. Baker, K. Gregory, J. E. Hill, K. Jahoda, P. Deines-Jones, Z. Prieskorn, and J. Swank. This work was performed in part under NASA contract NNG07EJ03C.

\section{Author Information}

\section{Kevin Black}

Rock Creek Scientific

Silver Spring, MD

and

NASA's Goddard Space Flight Center

Greenbelt, MD

Kevin Black began learning the craft of developing gas detectors while earning a $\mathrm{PhD}$ in experimental particle physics at Yale University. For nearly 20 years, he has been building gas detectors for astronomical applications at the Laboratory for High Energy Astrophysics.
References

1. E. Costa et al., An efficient photoelectric $x$-ray polarimeter for the study of black holes and neutron stars, Nature 411, pp. 662-665, 2001.

2. J. K. Black et al., X-ray polarimetry with an active-matrix pixel proportional counter, Nucl. Instrum. Meth. A 513, pp. 639-643, 2003. doi:10.1016/j.nima.2003.07.015

3 . R. Bellazzini et al., A sealed gas pixel detector for $x$-ray astronomy, Nucl. Instrum. Meth. A 579, pp. 853-858, 2007. doi:10.1016/j.nima.2007.05.304

4. J. K. Black, TPCs in high-energy astronomical polarimetery, J. Phys. Conf. Ser. 65 p. 012005, 2007. doi:10.1088/1742-6596/65/1/012005

5. J. K. Black et al., X-ray polarimetry with a micropattern TPC, Nucl. Instrum. Meth. A 581, pp. 755-760, 2007. doi:10.1016/j.nima.2007.08.144

6. T. Tamagawa et al., Development of gas electron multiplier foils with a laser etching technique, Nucl. Instrum. Meth. A 560, pp. 418-424, 2006. doi:10.1016/j.nima.2006.01.047

7. R. Bainbridge et al., Production testing and quality assurance of CMS silicon microstrip tracker readout chips, Nucl. Instrum. Meth. A 543, pp. 619-644, 2005 doi:10.1016/j.nima.2004.11.050

8. P. J. Serlemitsos et al., The X-Ray Telescope onboard Suzaku, Publ. Astron. Soc. Jpn 59, pp. S9-S21, 2007. 